\title{
Solid body rotation vibration
}

\author{
Kern E. Kenyon \\ 4632 North Lane, Del Mar, California 92014-4134, USA
}

\section{Keywords: Solid Body Rotation, Vibration}

\begin{abstract}
For solid body rotation of an incompressible fluid inside a vertical cylinder (open to air at the top with gravity acting down) it is proposed that if a small piece of fluid is displaced horizontally and radially by a short distance and then released, it will oscillate with a constant frequency as viewed in the rotating frame of reference. The oscillation frequency is derived to be equal to the basic frequency of the solid body rotation. Also it is hypothesized that a wave type of motion of the solid body rotation should be possible at the air/fluid interface. Experimental verification is needed for the conjectured vibration and wave motion of the solid body rotation.
\end{abstract}

\section{INTRODUCTION}

Solid body rotation is not found listed in the index of a typical fluid dynamics text. There are one or two exceptions ${ }^{[1]}$ On the other hand, the approach to solid body rotation, called spin-up, has inspired a whole book to be written. ${ }^{[2]}$

One guess as to why so little is printed about solid body rotation is that the easiest way to understand this rather simple steady fluid flow phenomenon is by making use of the centrifugal force, but that is taboo in classical physics.

Think of a vertical cylinder containing water that has been at constant rotation about its vertical axis for a long time until the water is at solid body rotation. Gravity acts down. An interesting feature of the solid body rotation is that the velocity increases linearly in the radial direction from the center of rotation whereas the pressure increases quadratically. Acquaintance with fluid boundary layers attached to solid surfaces might not necessarily lead one to expect this disparity in the decay rates for pressure and velocity. As an example, for steady flow past a cylinder the flow velocity decreases away from the cylinder like the inverse square of the distance by the standard theory, but the pressure field surrounding the cylinder is never presented for comparison ${ }^{[1]}$ Then there is the surface gravity wave, which typically has not been considered to be in the boundary layer category, where pressure and velocity are supposed to decay exponentially, and at the same rate, down from the equilibrium free surface, according to the way the subject is presently taught.

What is considered below, perhaps for the first time, is another aspect of solid body rotation. In the rotating frame of reference the flow does not move with respect to the observer. However, if a small piece of fluid is pushed horizontally and radially outward or inward a short distance, there will be a force that attempts to restore it to the initial position in both cases. Or if the piece of fluid is displaced and then left free to move, it should oscillate with a constant frequency. It will be shown below that this vibration frequency is that of the basic rotation rate.

\section{DERIVATION}

As a guide one of the available derivations of the Brunt-Vaisala frequency in the vertical direction will be adopted for the solid body rotation vibration in the horizontal direction ${ }^{[2]}$

At any particular initial location in solid body rotation, say ${ }^{r}$; , the horizontal forces on a fluid particle are balanced: the inward pressure force equals the outward centrifugal force. The horizontal pressure force arises hydrostatically from the height differences of the air/water interface. Let the particle be displaced radially outward by a small distance $\Delta r$ and then left alone. At the new position $r_{0}+\Delta r$ the inward horizontal pressure force is larger than it was at $r_{0}$ and by the amount 
$\omega^{2} \Delta r$, which equals the increased centrifugal force at the new location. For simplicity the constant density of the fluid is left out, and ${ }^{\omega}$ is the rate of the solid body rotation. Thus the particle will act according to the following equation of motion:

$\frac{d^{2} \Delta r}{d t^{2}}=\omega^{2} \Delta r$

Which is the harmonic oscillator equation with the restoring force on the RHS and the natural frequency being $\omega$

Similarly, if the particle were to be displaced from its initial position by the same small distance but radially inward, its centrifugal force would exceed the existing pressure force at the new location by the same magnitude as on the RHS of Eq. (1) and the sign would be reversed. Thus either way the particle is displaced, it feels a restoring force varying linearly with radial distance, like a spring. For best results the initial position of the particle that is to be displaced should not be too close to the cylinder wall, to the center of rotation or to the air/water interface.

\section{DISCUSSION}

What the above result means is that when the displaced particle has finished one complete vibration, the cylinder has made one complete rotation. If it can exist, the vibration would be "evidence" that the basic motion of the solid body rotation is stable, at least to this type of disturbance.

Verification of the solid body rotation vibration by experimental means may prove difficult mainly because of the rotating frame of reference involved. Perhaps it could be carried out using colored neutrally buoyant particles and finding some method of displacing them horizontally and radially. Extrapolating from the vibration of a single particle, it is conceivable that if many more particles were to be displaced just the right way all at the same time, a wave type motion might be generated. However, I am not aware that this suggestion has ever been made before. Has such a wave already been demonstrated in the laboratory?

None of the above conclusions requires that the basic vertical cylinder should have a horizontal cross-section that is circular. For example, a cylinder with a cross-section that is an ellipse, with the axis of rotation going through the center of the ellipse, would work just as well.

If the cylinder is closed at the top and filled with a compressible fluid, such as air, a vibrational motion of the solid body rotation should still be possible. In the steady state case the centrifugal force is balanced by a pressure force due to a linear increase of density with radial distance according to the perfect gas law, assuming constant temperature. Disturbance of the steady state for a small piece of air, radially inward or outward, will experience a restoring force analogous to a buoyancy force.

\section{REFERENCES}

[1] P. K. Kundu, Fluid Mechanics (Academic Press, San Diego, CA, 1990).

[2] H. P. Greenspan, The Theory of Rotating Fluids (Cambridge University Press, London, 1969).

[3] C. Eckart, Hydrodynamics of Oceans and Atmospheres (Pergamon Press, New York, 1960), p. 60 . 